\title{
Effect of alcohol and light on the retinal pigment epithelium of normal subjects and patients with retinal dystrophies
}

\author{
G B Arden, J E Wolf, F Singbartl, T E Berninger, G Rudolph, A Kampik
}

Applied Vision

Research Centre, Department of Optometry and Visual

Science, City

University, London

EC1V 7DD, UK

G B Arden

JE Wolf

University Eye

Hospital,

Mattildenstrasse 8,

Munich 80336,

Germany

F Singbartl

T E Berninger

G Rudolph

A Kampik

Correspondence to:

Dr G B Arden

Accepted 26 April 2000 g.arden@city.ac.uk

\begin{abstract}
Background-Light absorbed by photoreceptors causes oscillations in the voltage across the retinal pigment epithelium (RPE). This is the basis of the clinical test, electro-oculography (EOG). We have previously shown that alcohol causes a sequence of voltage changes which are so precisely the same as those caused by light that they must be produced by the same RPE machinery. There is good evidence that alcohol produces its effect by a direct action on the RPE. Consequently, in diseases associated with loss of photoreceptors, alcohol should continue to produce the voltage changes of the EOG unless secondary changes have occurred in the RPE.
\end{abstract}

Methods-The alcohol response in patients with retinitis pigmentosa (RP) was investigated using EOG.

Results-In no patient with RP was there any alcohol rise.

Conclusion-In patients with RP secondary abnormalities of function of the RPE must occur.

(Br F Ophthalmol 2000;84:881-883)

The sequence of events in the retinal pigment epithelium (RPE) following illumination has been analysed ${ }^{1-5}$ and has clarified the mechanism of action of clinical electro-oculography (EOG). ${ }^{6-9}$ When the dark adapted retina is illuminated a substance diffuses to the apical surface of the RPE and an intracellular "second

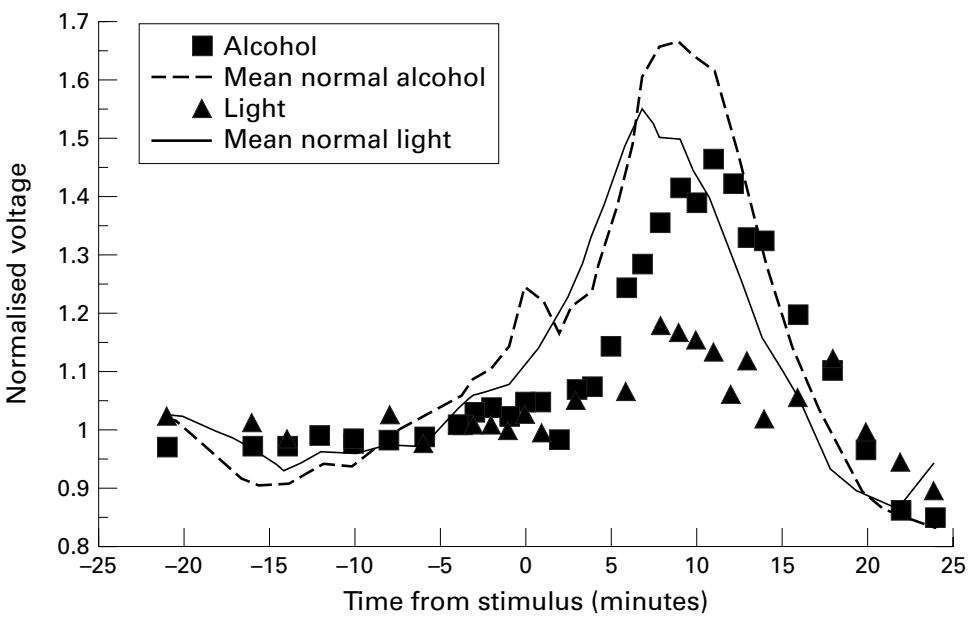

Figure 1 Effects of alcohol and light in a patient with myopic degeneration (symbols) compared with mean values of normal cases (lines). While the effect of alcohol is well preserved in the patient, the "light rise" is nearly absent. messenger" opens chloride channels in the basal RPE membrane, producing depolarisation and the increase in the transepithelial potential. If the retina cannot absorb light through the absence of photoreceptors, or if the subretinal space enlarges or the RPE is abnormal, this sequence cannot occur and the EOG voltage does not change with light. ${ }^{8-15}$ Without additional information it is impossible to distinguish between these various causes. We have recently found that the time course of EOG changes to light is exactly paralleled by a 10 second intravenous injection of $<1 \mathrm{~g}$ alcohol or by oral alcohol. ${ }^{15}{ }^{16}$ Since alcohol acts directly on the RPE, this may help to analyse EOG abnormalities in disease.

\section{Methods}

The well known technique of the $\mathrm{EOG}^{89}$ was used to measure standardised light and alcohol induced changes in a group of normal subjects and patients. The active leads were placed on the left and right temples. After 26 minutes of adaptation in darkness, during which time a stable baseline value of voltage was obtained, either the room lights were turned on $(50 \mathrm{~cd} /$ $\mathrm{m}^{2}$ with undilated pupils) or the subject drank $0.3 \mathrm{~g} / \mathrm{kg}$ alcohol diluted to $20 \% \mathrm{w} / \mathrm{v}$. Alcohol was given after fasting for $>10$ hours and was swallowed in 10 seconds. The light intensities used were deliberately submaximal to correspond with those in previous experimental work. ${ }^{15}$

\section{Results}

The lines in Figure 1 (reproduced from Miller and Steinberg ${ }^{10}$ ) show the mean EOG changes following exposure either to light or administration of oral alcohol. These were obtained in seven normal subjects with ages ranging from 22 to 70 . The mean voltage in the preceding 15 minutes is normalised to 1.0 on the ordinate. Note that the rises ( 1.55 for light, 1.65 for alcohol) are followed by a fall and (not illustrated) a series of oscillations. The value of the light rise is entirely consistent with the well known light intensity/response amplitude characteristic of the EOG, and was chosen to match the response (in darkness) to a moderate non-intoxicating dose of alcohol. The delay between the effect of light and alcohol is caused by the 3 minute delay before alcohol taken orally enters the systemic circulation. ${ }^{16}$ Figure 1 contains EOG data from a patient with myopic degeneration. He complained of loss of colour vision, which was progressive, and gave a history suggestive of night 


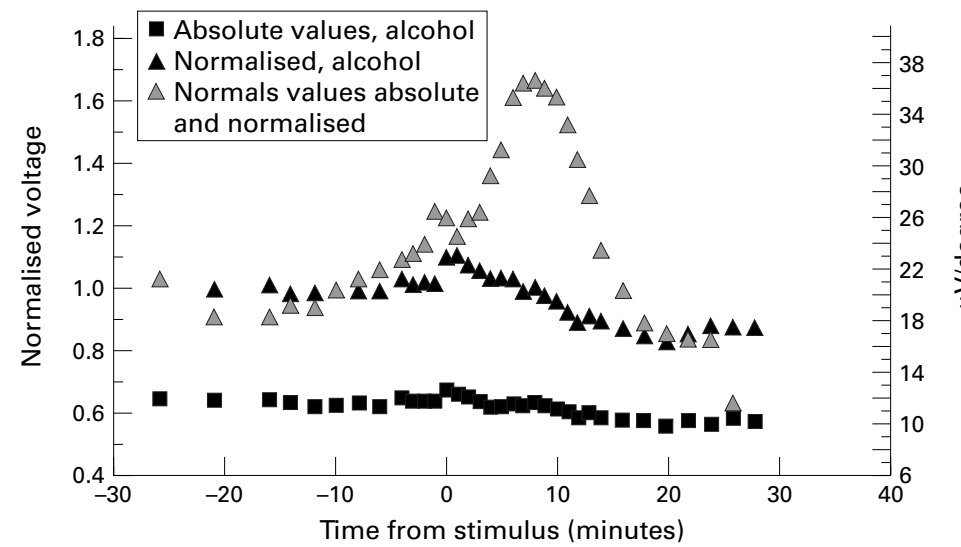

Figure 2 Average results for seven patients with retinitis pigmentosa. The EOGs were recorded with active and reference electrodes close to the right and left lateral canthi. The voltages were recorded to standard $30^{\circ}$ horizontal eye movements for sighted observers or extreme eye movements $\left(\sim 88^{\circ}\right)$ in blind observers. The absolute voltages expressed as microvolts/degree of eye rotation are shown on the right ordinate. The baseline voltage in normal subjects (grey triangles) is nearly double that in patients. The left hand ordinate shows data normalised to the mean during the 15 minutes before giving alcohol. The grey triangles show the normal alcohol rise and the solid triangles and squares the result in patients with retinitis pigmentosa. The alcohol rise is replaced by a delayed fall. The "peak" voltages are for times 4-8 minutes after the stimulus and the troughs were measured 16-22 minutes after the stimulus. Mean (SE) of patients' normalised responses baseline $=0.9978$ (0.0021); mean (SE) “peak" = $1.0126(0.029)$; mean $(S E)$ trough $=0.8214(0.043)$.

blindness. Field tests were normal. The electroretinogram (ERG) was well preserved, there being a slight reduction of amplitude and a generalised increase in peak times and a slight loss of sensitivity. The fundus photographs showed "laquer cracks" (a rupture in Bruch's membrane) just below the macula, with retinal and RPE thinning characteristic of myopia. The EOG light rise is effectively absent, but the alcohol rise is $92 \%$ of the mean normal, though the peak is delayed with respect to the normal.

In seven patients with retinitis pigmentosa (RP) very different results were found. The light rise of the ERG is known to be absent in $\mathrm{RP}$ so no data of standard clinical EOGs are presented. Figure 2 shows the mean alcohol results from the normal subjects (Fig 1) as open triangles. In RP the normal standing potential is reduced and the solid squares and grey triangles are plotted to the same absolute voltage scale (right hand ordinate). When the $\mathrm{RP}$ results are normalised (solid triangles, left ordinate) it can be seen that, following alcohol, the EOG voltages of the patients show a small progressive fall. The seven cases included two with dominantly inherited disease, one with Usher's syndrome, one X linked, and two simplex (we were unable to classify one case). All had complained of night blindness for a number of years. One simplex case was examined with an infrared scanning laser ophthalmoscope. There was a small perimacular area of RPE thinning, but the RPE was by no means clinically atrophic. This patient had normal multifocal (photopic) ERGs out to $24^{\circ}$ but in the $24-30^{\circ}$ region the response was extinguished. One dominant case had fields of $>90^{\circ}$ while two patients were blind and without any field. All the alcohol EOG results are very similar, as indicated by the statistics given in the legend to Figure 1. Even in the case with best preserved vision there was no "alcohol rise". Any possible correlation of the alcohol result with clinical or genetic features of RP requires a larger series (in preparation).

\section{Discussion}

Much evidence shows that alcohol and light effects only have a final common pathway within the RPE. ${ }^{16}{ }^{17}$ Thus, the alcohol rise is the same in darkness and in quite strong light although, if alcohol is already present in the bloodstream, a further dose of alcohol does not cause a change in RPE voltage. In the same way, the "light rise" to submaximal illumination is also independent of alcohol levels but is strongly inhibited by low levels of background illumination. ${ }^{16}$ Again, brief (1 minute) exposures to light or alcohol produce all the slow oscillatory voltage changes caused by prolonged stimulation, showing that the response is "triggered" or that, once the excitor substance is produced, it desensitises the RPE to further exposure. However, alcohol and light effects sum quantitatively even when one agent is given before or after the other. Thus, for submaximal stimulation, light and alcohol act almost independently. However, when larger amounts are given, the responses to each agent no longer sum but occlude, showing that they act through a final common pathway. ${ }^{16}$

In the myopic patient whose results are given in Figure 1 a markedly reduced light rise occurred with a (near) normal alcohol rise. This may be explained if the retinal degenerative changes of myopia have caused voids, reducing the concentration of the unknown "light substance" in the subretinal space while alcohol, diffusing from retinal and choroidal vessels as from an infinite source, is present in near normal concentration.

In all our cases of RP the normal "alcohol rise" was absent although the EOG voltage fell slowly in a manner reminiscent of the effects of acetazolamide or hyperosmolarity. ${ }^{913} 14$ It would appear that, following the death of rods in patients with $R P$, there are secondary changes to the RPE which result in the loss of the normal response to alcohol. It would be of interest to determine if this is so for all patients with RP including those who do not suffer from night blindness.

The absence of the normal alcohol response shows that alcohol cannot change the RPE conductances via an action on the surviving inner retina. However, in our patients with RP there are no or few rods, and it might be that alcohol acts directly on rods to cause the RPE changes. However, indirect evidence (summarised above) and experiments in vitro ${ }^{17}$ show that alcohol has a direct action on the RPE. The most obvious explanation for the results in Figure 2 is that the RPE mechanism which provokes the stereotyped increase in basal conductance has been lost in RP. The cause of the slower alcohol induced fall in RP remains to be explained. It should be noted that alcohol can reach the RPE from the choroidal circulation.

Since RPE conductance changes are associated with the transport of fluid and specific substances across the tissue, ${ }^{3}{ }^{10}$ secondary RPE changes could be associated with the loss of $\mathrm{RPE}$ function. This could explain the slow 
death of cones in dystrophies such as RP which are caused by point mutations in the gene coding for rhodopsin. ${ }^{18}$ Unless these changes are reversible, our findings could have implications for proposed schemes of retinal rescue since the retina is dependent on the normal functioning of the RPE.

1 Linsenmeier RA, Steinberg RH. Origin and sensitivity of the light peak of the intact cat eye. F Physiol 1982;331:653-73.

2 Miller SS, Edelman DJ. Active ion transport pathways in the bovine retinal pigment epithelium. F Physiol 1990;424:283-

300 .

Miller SS, Lin H. $\left[\mathrm{K}^{+}\right]_{0}$-induced changes in apical membrane voltage modulate $\mathrm{pH}$ in frog retinal pigment epithelium. Invest Ophthalmol Vis Sci 1989;130(Suppl) 168.

4 Steinberg RH, Linsenmeier RA, Griff ER. Retinal pigment epithelial cell contributions to the electroretinogram and electrooculogram. In: Osbourne NN, Chader GJ, eds. Progress in retinal research. New York: Pergamon, 1985 33-66.

5 Steinberg RH, Linsenmeier RA, Griff ER. Three light evoked responses of the pigmented epithelium. Vision Res 1983;23:1315-23.

6 Arden GB, Kelsey JH. Changes produced by light in the standing

7 Arden GB, Kelsey JH. Some observations on the relationship between the standing potential of the human eye and she bleaching and regeneration of visual purple. 7 Physiol 1962;161:205-26.
8 Arden GB, Barrada A. An analysis of the electroArden GB, Barrada A. An analysis of the electro-
oculograms of a series of normal subjects. Brf Opthalmol oculograms of a seri

9 Kolder H. Electro-oculography. In: Heckenlively J, Arden GB, eds. Principles and practice of clinical electrophysiology of vision. St Louis: Mosby Year Book, 1991:310-3.

10 Miller SS, Steinberg RA. Active transport of ions across the frog retinal pigment epithelium. Exp Eye Res 1977;25:23548 .

11 Gallemore RP, Steinberg RH. Effect of DIDS on the chick retinal pigment epithelium II: Mechanism of the light peak and other responses originating at the basal membrane. $f$ and other responses origina

12 Stavrou P, Good PA, Broadhurst EJ, et al. ERG and EOG abnormalities in carriers of X-linked retinitis pigmentosa. Eye 1996;10:581-9.

13 Pinckers A, Cuypers MH, Aandekerk AL. The EOG in Best's disease and dominant cystoid macular dystrophy (DCMD). Ophthalmic Genet 1996;17:103-8.

14 Miyake Y, Horiguchi M, Suzuki S, et al. Electrophysiological findings in patients with Oguchi's disease. Fpn F Ophthalmol 1996;40:511-9.

15 Arden GB, Wolf JE. Alcohol mimics the effect of light on the human electrooculogram (EOG) F Physiol 1999;517:P93.

16 Arden GB, Wolf JE. The human electro-oculogram: interaction of light and alcohol. Invest Ophthalmol Vis Sci 2000 (in press).

17 Bialek S, Quong JH, Yu K, et al. Non steroidal antiinflammatory drugs alter chloride and fluid transport in bovine retinal pigment epithelium. Am f Physiol 1996; 270C: $1175-89$.

18 Moore AT, Fitzke FW, Kemp CM, et al. Abnormal dark adaptation kinetics in autosomal dominant sector retinitis pigmentosa due to rod opsin mutation. Br $\mathcal{F}$ Ophthalmol pigmentosa due to 